\title{
A study of workload units in five microbiology laboratories
}

\author{
RY CARTWRIGHT, ${ }^{*}$ JOAN R DAVIES, $\uparrow$ C DULAKE, $\dagger$ RJC HART, $\ddagger$ CA MORRIS, $\S$ \\ PJ WILKINSON" \\ From the Public Health Laboratories, *St Luke's Hospital, Guildford, †William Harvey Hospital, \\ Willesborough, Ashford, Kent, $\ddagger$ Heavitree, Exeter, §Royal Shrewsbury Hospital, "Derriford Hospital, \\ Plymouth, and the $\llbracket$ Public Health Laboratory Service Board, Head Office, Colindale, London NW9
}

SUMMARY A study of a modified Canadian unit system of measuring laboratory workload was $\infty$ undertaken in five joint Public Health Laboratory Service and hospital microbiology laboratories. Ten percent of the specimens received over six months were sampled, the number of units expended on each was recorded, and the results were analysed on a central computer. The process of gathering information in the absence of laboratory computers was time consuming and, despite careful planning, differences were found in the recording practices of the laboratories. The analysis of results did not lead to major changes in data gathering techniques because the same information about laboratory workload could be obtained by collecting numbers of clearly defined specimens. Analysis of workload units could be useful for particular purposes, such as comparing differences between laboratories using different techniques for the same investigation or assessing the possible benefits of automation. It must be appreciated, however, that workload units are measures of quantity not of laboratory performance.

For some years there has been dissatisfaction with the methods of assessing laboratory work. They have been criticised as being both inaccurate and uninformative. Attempts to improve the methods have led in Britain to redefinitions of requests and specimens and in North America to the use of workload units. These different approaches may be a reflection of the different methods of financing laboratory work.

The complexity of microbiological investigations compared with the more stereotyped and mechanised techniques used in haematology and chemical pathology make for more difficulty in workload measurement. In general, the information required of a microbiology laboratory is the total workload, the distribution of specimen types, and the work entailed in the examination of each type. It may also be desirable to record the source of specimens and to provide a means of costing the examination. None of these measurements is of any value unless the method of calculating the workload is reliable, reproducible, and provides for uniformity between different types of specimen and between different

Accepted for publication 9 October 1984 laboratories. Within the laboratory this information is necessary for the best allocation to be made of manpower and space. It should be sufficiently precise to enable assessment of trends in workload leading to the review of methods so as to provide, for example, information on the cost effectiveness of automation.

Health authorities need information to allocate resources between laboratories more rationally. If $ᄋ$ costing of laboratory services to particular users, such as individual clinical disciplines, can be made sufficiently accurate, realistic forecasts of the $\frac{T}{0}$ resource requirements of the laboratory can be made to accommodate changes in clinical workload. $N$ Accurate statistics of workload would be of particu- $N$ lar value to the Public Health Laboratory Service (PHLS), in which there are a number of closely $\omega$ comparable laboratories, because they would provide opportunities for the evaluation of different $\stackrel{\varrho}{\subseteq}$ methods and techniques as well as providing the administrative information already discussed.

Before the present study, discussion in Britain 1 about the Canadian unit system ${ }^{2}$ of measuring workload led to an experimental assessment of unit work- $\frac{\rho}{\Phi}$ load measurement in pathology by the South West- $\stackrel{\mathbb{D}}{\square}$ ern Regional Health Authority (SWRHA). A 
number of combined PHLS and hospital laboratories were involved in the application of this project to microbiology, the funding of which ceased after much preliminary work had been done. This information was made available to us, and a six month pilot study of a modified Canadian unit system of workload measurement was carried out in five laboratories, each of which provided a diagnostic service and examined food and other environmental specimens. The units were based on technical time only; no consideration was given to overheads, the employment of other staff, or the capital and revenue costs of equipment. Each laboratory collected its own data, which were analysed by the computer services department of the PHLS.

\section{Methods}

The participating laboratories all performed a wide range of microbiological investigations employing commonly used manual methods at the time of the survey, which were broadly comparable for each. Two of the laboratories examined about 60000
Table 1 Examples of allocation of workload units (WLU) to laboratory tests

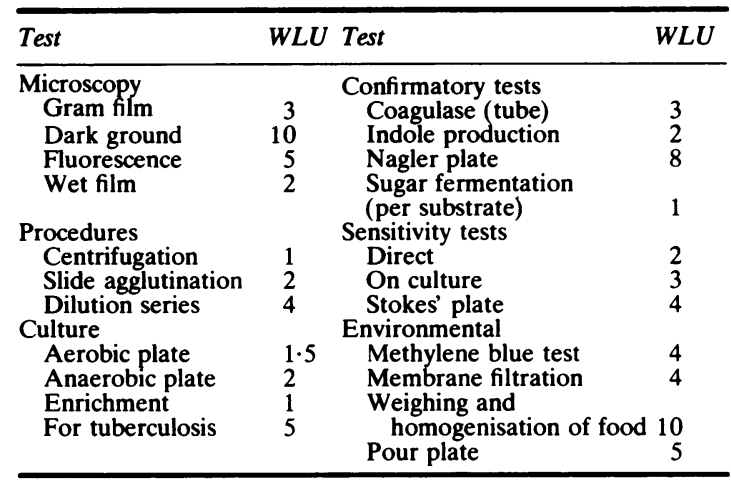

specimens a year, two examined about 100000 , and one 140000 .

The examination of a specimen implies the performance of a number of laboratory manoeuvres

\section{P. H. L. EXETER WS/241/50/01}

\begin{tabular}{|c|c|c|c|}
\hline CLERICAL & 037 & & \\
\hline VISUAL EXAMINATION & 262 & & \\
\hline HOMOGENISATION & 093 & & \\
\hline LIQUEFACTION/DIGESTION & 131 & & \\
\hline METHYLENE BLUE STAIN & 155 & & \\
\hline DIRECT BLOOD AGAR $\mathrm{O}_{2}$ & 043 & & \\
\hline$" \quad " \mathrm{CO}_{2}$ & 044 & & \\
\hline$" \quad " \quad \mathrm{AnO}_{2}$ & 044 & & \\
\hline CHOCOLATE AGAR $\mathrm{CO}_{2}$ & 044 & & \\
\hline CLED & 043 & & \\
\hline MoC & 043 & & \\
\hline NALIDIXIC BLOOD AGAR & 044 & & \\
\hline MANNITOL SALT AGAR & 043 & & \\
\hline$S A B C 27^{\circ}$ & 043 & & \\
\hline$" \quad " 37^{\circ}$ & 043 & & \\
\hline SUCROSE BROTH & 045 & & \\
\hline MYCOPLASMA MEDIUM & 044 & & \\
\hline BROTH CULTURE & 045 & & \\
\hline GRAM (SPECIMEN) & 153 & & \\
\hline ZN (SPECIMEN) & 161 & & \\
\hline EOSINOPHIL STAIY & 154 & & \\
\hline CENTRIFUGE & 034 & & \\
\hline WET PREP (DIRECT) & 159 & & \\
\hline READ DIP SLOPE & 054 & & \\
\hline WET PREP (SPUN DEPOSIT) & 159 & & \\
\hline SENSITIVITY (DIRECT) $37^{\circ}$ & 223 & & \\
\hline$" 30^{\circ}$ & 223 & & \\
\hline$"$ (CULTURE) $37^{\circ}$ & 224 & & \\
\hline
\end{tabular}

SPUTA AND SPECIMENS FOR T.B.

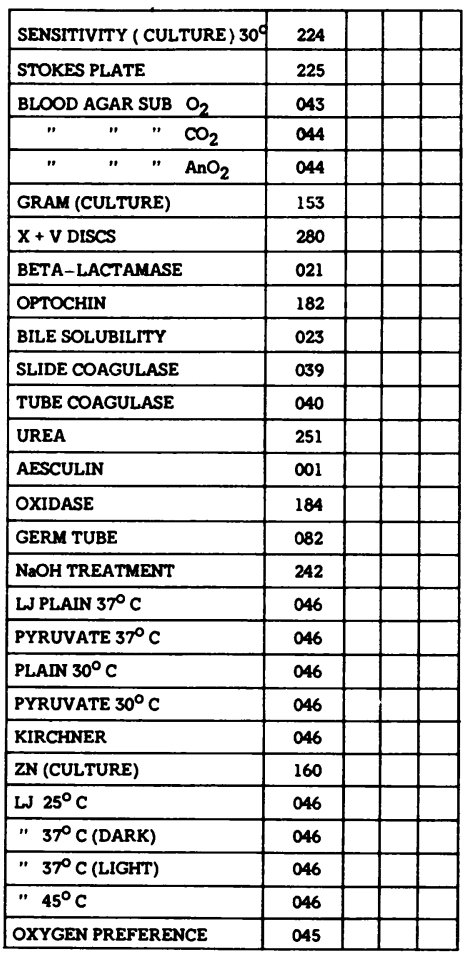

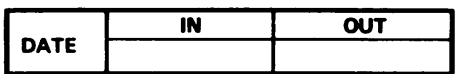

\section{LAB. NUMBER}
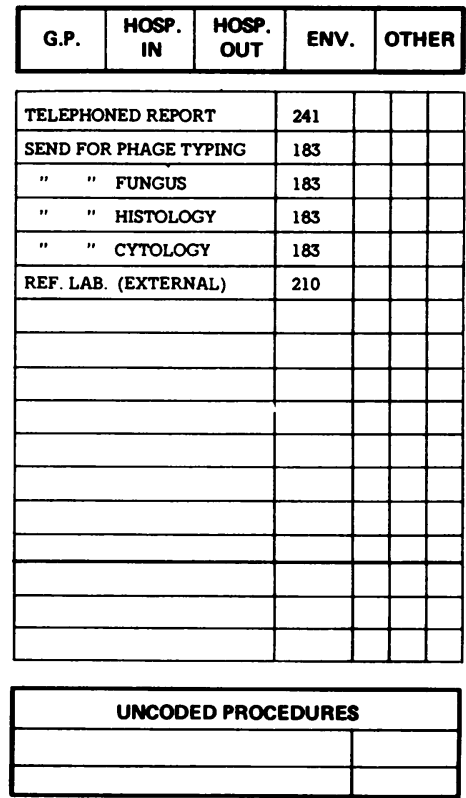

Fig. 1 Example of a backing sheet. 
(tests), which may involve microscopy, culture on a variety of media, subculture, characterisation of organisms, assessment of antibiotic sensitivity, the quantitative assessment of antibodies to a number of antigens, and so on. A list of all tests performed by the participating laboratories was prepared and each test was allocated a three digit code, a name, and a score of notional workload units (WLU). These scores were allocated by one of three methods: $(a)$ acceptance of the units given in the Canadian system $^{2} ;(b)$ adoption of the unit value worked out in the South Western Regional project; and (c) preparation of new scores for tests or methods for which no unit values were available.

When method 3 was used the tests were carried out in at least two laboratories by several members of staff with differing degrees of experience. The unit value for each test was the average number of minutes required to carry it out. The scores, however derived, were agreed by the participating laboratories. Where different laboratories used different methods to perform a test, an appropriate score of WLU was allocated to each method. Examples of the WLUs are shown in Table 1.

Specimens were classified into 14 groups and for each group an appropriate backing sheet was designed listing the tests likely to be used (for example, see Fig. 1). Additional sheets were used if further tests were necessary. The specimen categories were: urine, upper respiratory, lower respiratory, genital, wound, blood culture, cerebrospinal fluid, faeces, mycology, tuberculosis, general bacteriology, serology (including viral serology), virus isolation, and environmental microbiology (including food, milk, water). Specimens received for external quality assurance were included in the appropriate categories. Throughout the study all specimens were allocated laboratory numbers by the system normally used in the laboratory. The request form accompanying every 10th specimen in each numbering series was attached to the appropriate backing sheet. Members of the laboratory staff ticked the appropriate tests

Table 2 Comparability of laboratories: specimens and workload units (WLU)

\begin{tabular}{lclll}
\hline $\begin{array}{c}\text { Laboratory Total } \\
\text { specimens } \\
\text { per annum* }\end{array}$ & $\begin{array}{l}\text { Specimens } \\
\text { assessed }\end{array}$ & $\begin{array}{l}\text { Mean WLU } \\
\text { per specimen }\end{array}$ & $\begin{array}{l}\text { Total annual } \\
\text { WLU } \\
\text { (thousands) }\end{array}$ \\
\hline A & 58640 & 2932 & $19 \cdot 53$ & 1145 \\
B & 61480 & 3074 & $13 \cdot 19$ & 811 \\
C & 93840 & 4692 & 16.09 & 1509 \\
D & 96800 & 4480 & $15 \cdot 72$ & 1522 \\
E & 131460 & 6573 & 13.88 & 1825 \\
\hline
\end{tabular}

*Estimated from a $10 \%$ sample over 25 weeks. on the backing sheet while the specimen was under examination.

When work on each specimen in the survey was complete and the report signed, the backing sheets were separated and sent in weekly batches to the computer services department of the PHLS. There the scores of WLU were added to the sheets and the information processed on a CTL 8046 computer (Computer Technology Ltd, Hemel Hempstead, England). None of the participating laboratories had a local computer facility.

\section{Results}

All five participating laboratories provide a general microbiology diagnostic service predominantly for the examination of clinical specimens, of which about $70 \%$ were from hospitals and the remainder from other sources; these included a small number of non-clinical specimens such as milk, water, and food. Table 2 shows for each laboratory the estimated annual workload (calculated from the 25 week study period) expressed in terms of specimens and units. The proportion of different categories of specimen sampled and workload units generated by them in each laboratory is shown in Fig. 2. As expected, there was some variation between laboratories but the patterns showed no appreciable differences and all five laboratories could be regarded as broadly comparable in the source and type of specimen and proportion of samples in each defined category.

For each category the mean workload units per specimen was calculated for each laboratory and for all laboratories. Fig. 3 shows the variation above and below the group mean. The specimens are divided into three groups: $(a)$ those in which there is little variation between laboratories - for example, upper respiratory and urine; $(b)$ those in which differences are moderate-for example, wound, faeces, serology; and (c) those showing wide variation-for example, lower respiratory, blood cultures, and mycology.

An attempt has been made to explain the more extreme differences above or below the group mean and some of the probable or possible reasons are listed below.

1 Lower respiratory tract. Laboratory C, unlike the others, routinely examined all sputum samples from new patients for the presence of acid fast bacilli. The other laboratories entered all specimens examined for acid fast bacilli (whether or not specifically requested) in the category tuberculosis.

2 Blood cultures. Some laboratories scored each inoculated broth in a blood culture set as one 
A study of workload units in five microbiology laboratories

(A)

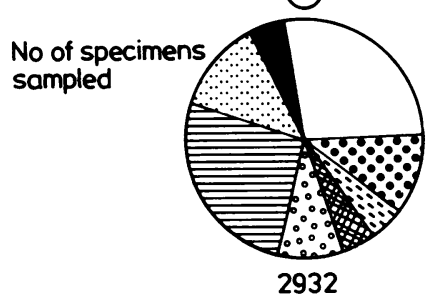

2932

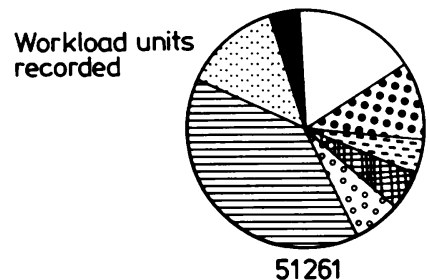

51261
(B)

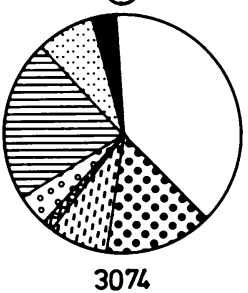

3074
(C)

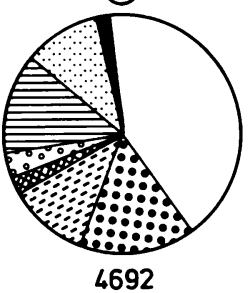

4692
(D)

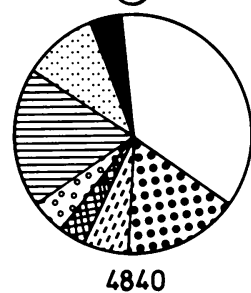

4840

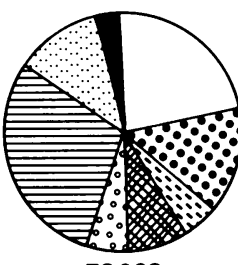

76093
(E)
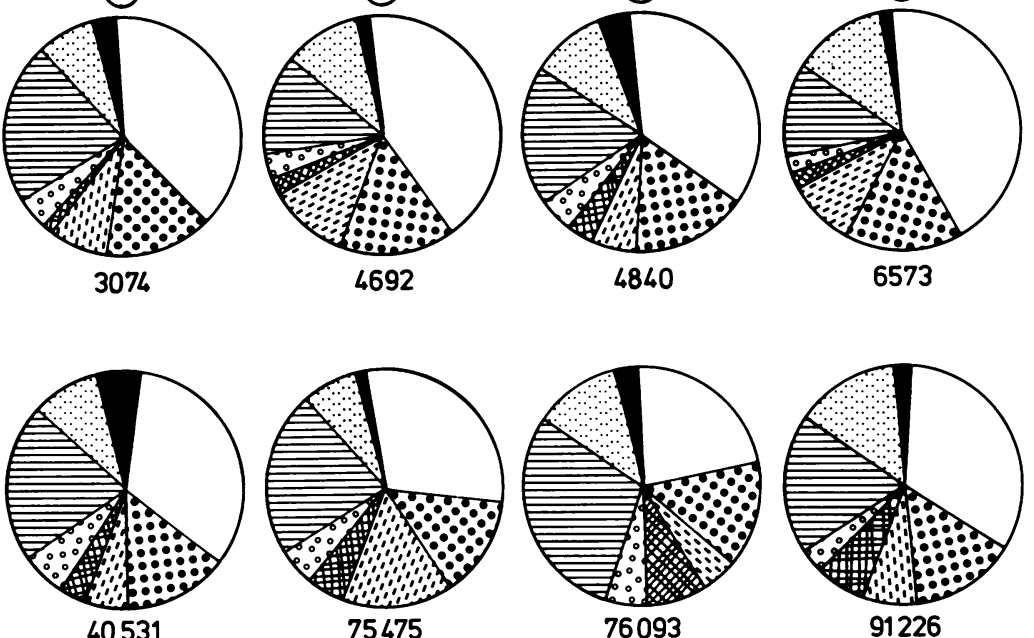

40531

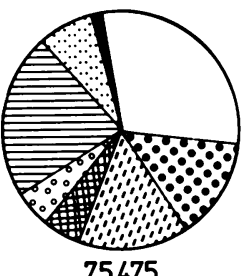

75475

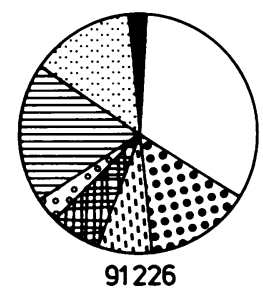

$\square$ Urines
$\begin{aligned} & \text { Wound and } \\ & \text { genital }\end{aligned}$

Fig. 2 Specimens sampled and workload units generated in each laboratory.
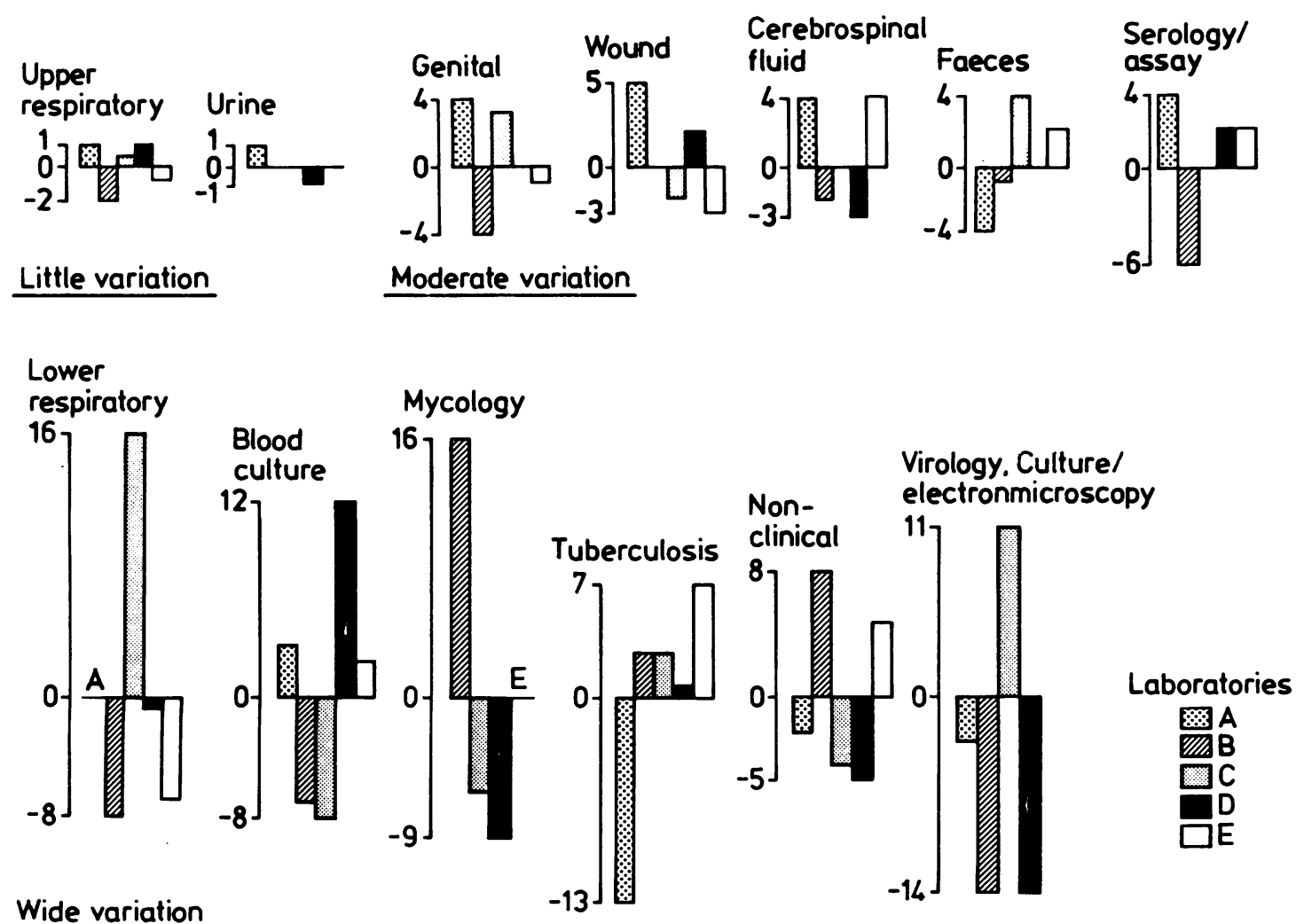

Wide variation

Fig. 3 Variation between laboratories in mean workload units per specimen. Laboratory A did not undertake mycology and laboratory $E$ did no virology (culture or electron microscopy). 
Table 3 Ranking of laboratories by total specimens, total workload units (WLUs) and WLU/specimens

\begin{tabular}{lll}
\hline Total specimens & Total WLUs & Mean WLU/specimen \\
\hline A (lowest) & B & B \\
B & A & E \\
C & C & D \\
D (highest) & D & C \\
E & A \\
\hline
\end{tabular}

specimen while others scored all the broths inoculated from the same blood as a single specimen. Laboratory $\mathrm{D}$ had a relatively high isolation rate from blood cultures necessitating on average more tests for identification and antibiotic sensitivities than the others. This was probably a reflection of samples from specialist units (renal transplant and immunocompromised patients).

3 Mycology. Laboratory B, unlike the others, was unique in employing a scientist with a substantial commitment to mycology. Laboratory A did not undertake mycology.

4 Tuberculosis. Laboratory A did the preliminary work on specimens for isolation of Mycobacterium tuberculosis and passed all suspect cultures to a separate reference laboratory nearby.

5 Non-clinical. Laboratory B provided a "flight meal" test service for a major airport.

6 Virology. Laboratory E did not offer a virus culture or electron microscopy service. A higher proportion of neutralisation tests was performed by laboratory $\mathrm{C}$ compared with laboratories $\mathrm{A}$ and $\mathrm{D}$. This difference reflects a local epidemic of virus associated illness.

Table 3 lists laboratories in rank order according to the total number of specimens, the total workload units, and the mean number of workload units per specimen. The order is closely similar for total specimens and total workload units but there are appreciable changes in ranking when workload units per specimen are considered.

Additional data collected but not presented here included the following: (a) source of specimen (general practitioner, hospital inpatient, hospital outpatient); (b) the mean number of specimens per family doctor, per 100 deaths or discharges, per 100 outpatients; $(c)$ distribution of workload throughout the week.

\section{Discussion}

A realistic and accurate assessment of laboratory workload is necessary for effective distribution of resources between laboratories and for good laboratory management. The extent to which details are collected and analysed will depend on the purposes for which they are required and whether the information gained justifies the expense of collecting it. It is well recognised that counting requests alone is a crude and often misleading measure of workload. This is particularly the case when an attempt is made to compare the workload in different pathology disciplines, some of which use automated techniques and others use predominantly manual methods. Improvements have been attempted by weighting the requests by average test to request ratios. A more detailed but time consuming method of assessing laboratory workload, such as the Canadian system, records the units of work for each test or procedure on each specimen. The Körner report ${ }^{3}$ concluded that the Canadian system did not overcome the problems inherent in a weighted request number count and recommended that numbers of requests only should be collected by health authorities for the Department of Health and Social Security. The decision whether to gather any additional information should be left to individual health authorities. The debate on obtaining comparative statistics about activity and the use of resources has continued in Britain with the publication ${ }^{4}$ of performance indicators over a range of health district functions.

This study using a modified Canadian workload unit system has compared five combined public health and hospital microbiology laboratories. We appreciated from the outset that the information would relate only to the work associated with the specimens examined. It did not include media preparation and wash up, administration, research and development, internal quality control, consultation time, or training. The measurements were all of quantity and not quality and thus the study assessed workload and not performance.

The determination of the unit values chosen was affected by the mechanisation of tests, on whether tests were performed individually or in batches, and on the experience of the staff. Some procedures were given alternative unit values depending on the methods used. When the results were analysed it became apparent that the values assigned for certain procedures required revision as the total workload for some specimen groups was not proportional to the actual time spent on those specimens. This was particularly evident for urine, which comprised the greatest number of specimens examined in each laboratory. Minor errors in the unit values of even small procedures were amplified out of proportion and thus distorted the pattern of the workload of the laboratory. We also realised that accuracy and time taken in recording workload could be improved by grouping certain routine procedures. This would 
also permit adjustment of the group unit values to a more realistic figure. If, for example, all specimens of urine had a microscopical examination, protein estimation, and culture, these tests recorded individually might qualify for three units but if grouped might attract only two units.

The particular problem in microbiology, unlike other laboratory disciplines, is that the work required on a specimen cannot necessarily be determined when the specimen is received. The results of initial tests may determine the next series of tests. The information which is provided with a specimen may also affect the tests performed. The examination of specimens from contacts, or for confirming the absence of an organism after recovery from an infection, may be more limited than the examination of a similar specimen when the pathogen is unknown. On the other hand, when it is known that a patient has travelled abroad a more extensive examination may be required. Even the final report on a specimen is not an accurate reflection of the work undertaken-for example, a negative result on a faeces specimen may have been produced only after extensive biochemical and serological testing of some non-lactose fermenting organisms.

Because an accurate estimation of workload on an individual specimen cannot be obtained from either the request or the report it is necessary to record the actual work undertaken. Logistically, it was not possible to do this for every specimen, so a $10 \%$ sample was used in this study. This produced a sample size of 3000 to 6000 specimens per laboratory and was adequate to give an overall estimate of laboratory workload. Detailed information on specimen groups with only small numbers-for example, cerebrospinal fluid-would require all specimens in this group to be included in the analysis. It has been suggested that the introduction of computers would enable the workload associated with each specimen to be measured. This would be true only if every test undertaken was entered into the computer. It is common practice, however, only to enter results and not all the steps entailed in reaching them; similarly several bacterial colonies may be examined but only the presence of any recognised pathogen is recorded.

The regular collection of workload data relies on the cooperation of the staff. All the staff cooperated willingly in this study, encouraged by knowing that it was for a limited period only. The gathering of data did mean a great deal of extra work for them, and the accuracy of workload measurement by this method over longer periods might well suffer because of this.

The results of this study reflect the broadly similar nature of the five collaborating laboratories. All were area public health laboratories receiving a similar range of specimens, most of which were examined by similar techniques. Under these circumstances the workload is related to the total number of specimens received and further refinements of measurement would be unlikely to yield significant additional information. Table 3 shows that whether laboratories are ranked by total specimens received or by total workload units recorded, the order is not significantly different. Only if a laboratory received a substantial proportion of specimens requiring examination by complex techniques would measurement of the work actually done reveal a higher workload. Laboratory $A$ had a higher proportion of virology specimens than other laboratories, which accounts for the fact that it had the highest mean workload unit to specimen ratio (Table 3), although it had the lowest number of specimens.

We did not consider that analysis of workload units by source of specimen or by deaths, discharges, or outpatient attendances would yield any more useful information than was obtainable by counts of specimens. Although an analysis of the distribution of work throughout the week is not recorded here, it was apparent that different and misleading information could be obtained depending on whether the work was related to the day the specimen was received, the day most of the bench work was done, or the day the result was issued. This is in sharp contrast to the situation in biochemical and haematological laboratories, where the workload pattern can be clearly seen because the tests are usually completed and reports issued on the day specimens are received. The need to incubate cultures for $18 \mathrm{~h}$ or longer means that estimates of the work undertaken on different days will differ depending on the recording days used. This needs to be taken into account if the information is to be used-for example, to assess staffing requirements for weekend work.

What then is the value of using workload units if they do not immediately answer all managerial and administrative questions? One possible use is to compare the amount of work different laboratories devote to the examination of a particular type of specimen. Although most of the wide variations identified in this study resulted from a failure to define terms sufficiently accurately, they did show some real differences in practice. Another potential use is to obtain a quantitative estimate of the bench time taken in an examination by manual methods to assess the likely advantage of automation. Although other considerations such as capital and revenue costs and speed and accuracy of reporting results 
would be included in such an assessment, it is important to estimate what manpower resources might be liberated.

The PHLS has not continued with collecting workload data but uses specimen counts on one selected day every month. The specimens are divided by type and source. This study indicates that the impact of any important changes could be examined by measuring the workload entailed.

It must be clearly understood by all who use or wish to use workload units that they measure the work actually done, not what should be done. Quantity and quality are not synonymous. It may be misleading to assume that a laboratory which shows a low workload unit per specimen ratio is managed in a more efficient, selective, or cost effective way. It may simply be that such a laboratory is underresourced to the extent that it is forced to provide a suboptimal service.

We are grateful to the South Western Regional Health Authority for making the results of their preliminary study available to us. Dr GT Mills and $\mathrm{Mr}$ AD Kilburn generously shared their expertise and experience with us and gave invaluable advice. Our head medical laboratory scientific officers, Messrs JP Alexander, GF Down, TA Ford, R Human, DA Porter, and Dr RA Bassett played important parts in organising the trial.

\section{References}

1 Morris CA. Trends in microbiology work-loads in the National Health Service: England and Wales (1968-79). Health Trends 1981;13:8.

${ }^{2}$ Statistics Canada. Canadian schedule of unit values for clinical laboratory procedures. Ottawa: Statistics Canada, Health Division, 1978.

${ }^{3}$ National Health Service/Department of Health and Social Security. Steering Group on Health Services Information. $A$ report on the collection and use of information about hospital clinical activity in the National Health Service (Chairman Mrs E Körner). London: HMSO, 1982.

+ Department of Health and Social Security. Health services management performance indicators. London: DHSS, 1983. (DHSS Health Notice (83) 25).

Requests for reprints to: Dr RJC Hart, Public Health Laboratory, Church Lane, Heavitree, Exeter EX2 5AD, England. 